\title{
Environmental management and numerical models: examples from long-term ecological research on a real case study
}

\author{
C. Solidoro, G. Cossarini \& D. Melaku Canu \\ Istituto Nazionale di Oceanografia e di Geofisica Sperimentale OGS, \\ Italy
}

\begin{abstract}
Numerical models provide a framework for the integration and synthesis of existing knowledge about an ecosystem, and might offer important contributions for understanding the scale of human disturbance and the potential effectiveness of restoration action. In addition they provide the quantitative knowledge which is required for evaluation, at ecological and economic levels, of consequences of the implementation of possible alternative scenarios of policy options. Coastal areas and estuaries are particularly important sites, since they are very sensitive to anthropogenic impact and are very valuable. We present applications of a long-term modeling study on the Lagoon of Venice, which is the largest Italian lagoon. A suite of models, including a 3D fully coupled transport-water quality model, has been developed, validated against a substantial amount of real world data, and used to investigate nutrient cycles, primary production and water quality status. The model has then been used to analyze the effects of different scenarios of loading from the drainage basin, and to solve the inverse problem of the identification of Maximum Permissible Load that is compatible with the predefined Water Quality Target. Other applications include the integration of the model with a model for the growth and population dynamic of clams, and the identification of economically efficient and ecologically sound policies for exploitation of this renewable resource, in agreement with paradigms from sustainable developments. Finally, the model has been modified, and applied to derive a contribution to the evaluation of effects of multiple temporary closures of lagoon inlets on the water quality of the lagoon.

Keywords: ecological modelling, water quality, lagoon of Venice, macroalgae, clams, MOSE, maximum permissible loads, ICZM, LTER.
\end{abstract}




\section{Introduction}

The request for model applications to ecological and environmental management problems has been steadily increasing during the last few years, and nowadays the use of ecological models is advocated in the majority of research programs. Models are included in major IGBP programs, which aim to assess the possible impact of global changes on global, regional and local systems, as well as in environmental impact assessment of specific local interventions and management problems. In fact, besides providing a theoretical frame within which to integrate available experimental information and ecological theories, mathematical models often represent the only way of assessing the effectiveness of alternative management options and forecasting their consequences on a given ecosystem and, therefore, they might give a substantial contribution to the so-called 'decision support systems'.

In this paper we briefly present selected examples of our applications of ecological models to management problems in the lagoon of Venice. This site has been the object of a large number of studies, including a long-term ecological modelling study, and can be considered as a natural lab, in which to test both new scientific methodologies and implementations of new management interventions. The problems were dealt with by using coupled transport-reaction models. The first two case studies represent specific applications concerning two general problems, i.e. species harvesting and the management of the Nitrogen and Phosphorus loads. The third case study concerns a very site-specific, but extremely important problem, i.e. the assessment of the environmental impact of the closures of the lagoon inlets.

\section{The case study: the lagoon of Venice}

The lagoon of Venice, which is located in the Northern Adriatic Sea, is the largest Italian lagoon, covering an area of about $500 \mathrm{~km}^{2}$. Its average depth is less than $2 \mathrm{~m}$, but its morphology is characterized by the presence of large shallow areas and by a network of deeper channels. As can be seen in Fig. 1, three narrow inlets connect the lagoon to the Adriatic Sea. The lagoon is conventionally partitioned into three sub-basins, which are separated by two watersheds, along which the tidal velocity are low. The lagoon of Venice hosts highly valuable typical habitats, as well as several economic activities which depend upon the ecosystem health, such as fisheries, recreational activities and tourism. At the same time, the presence of other important economic activities negatively affects the quality of this environment. Among others, the port activity and the industrial ones, which are still concentrated in the area of Porto Marghera, see Fig. 1, should be mentioned. In addition, the lagoon receives the discharge of a drainage basin equivalent to 4,000,000 inhabitants, which conveys into the lagoon approximately $0.5 \times 10^{6} \mathrm{Kg}$ of Nitrogen and $1.5 \times 10^{6} \mathrm{Kg}$ of Phosphorous per year (Solidoro et al. [1]). 


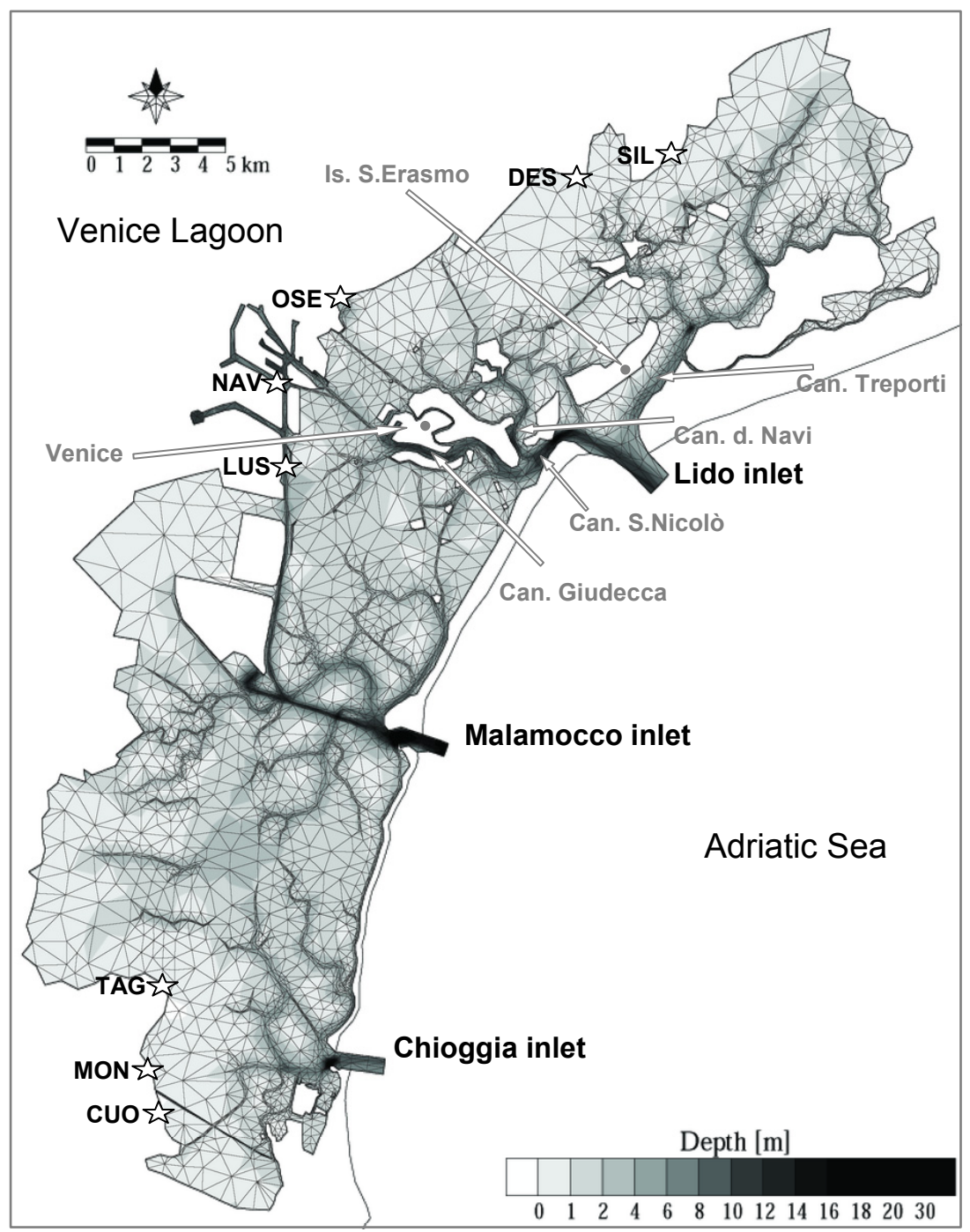

Figure 1: $\quad$ Schematic bathimetry of the lagoon of Venice, as obtained by using the grid of the numerical model, superimposed. Channels can be distinguished by the darker color and by the fact that they are represented by smaller elements. The white stars at the inner border of the lagoon mark the input points of major tributaries (from Solidoro et al. [2]).

The lagoon is a paradigmatic example, and the result of human interventions which hinder the natural ecological succession of the lagoon in either a marine or land environment (Ravera [3]). The fight is still going on, and several interventions are presently being discussed, which might have a substantial effect on the nutrient cycling and on the eutrophication of the lagoon. Among these one 
can remember: the temporary closure of inlets in concomitance with exceptionally high tides, in order to prevent the flooding of large parts of the city, which might reduce exchange with the sea; the setting up of intensive aquaculture of the bivalve Tapes philippinarum in selected areas, which might solve some of the problems caused by the unregulated access to this resource, but could also enhance nutrient recycling and locally deplete the planktonic stocks; the plan for the reduction of the nitrogen loads from the tributaries.

\section{The models}

Coupled transport-reaction models are made essentially of two parts. The first one is the submodel which describes the transport of dissolved substances while they react with each other. The second one describes biogeochemical reactions.

In the lagoon of Venice, residual currents (i.e. currents averaged over a tidal cycle) are almost negligible everywhere, which means that a drifter would follow a roughly elliptical path and return approximately to its release point. This implies that the area when subjected to diffusion can be approximated by an ellipse, and that, insofar as one is not interested in processes shorter than the tidal cycle and in the effect of actual meteorological forcing, transport processes can be approximated by anisotrophic eddy diffusion, with tensor coefficients estimated once for all from a climatological current field. This approximation has been adopted in some of the applications presented here, where the focus was on the seasonal scale. In those cases a finite difference parameterization of the transport equation was implemented. Details on this scheme can be found in Solidoro et al. [1] and references therein. A finite element discretization of the shallow water equation was instead used for the analysis on temporary closure of the inlets, where the time scales of interest were shorter than the tidal cycle, and effects of realistic forcing (wind and tide) were important. Details on this scheme can be found in Umgiesser et al. [4].

The biological module describes the cycles of nitrogen, phosphorus and carbon and their interactions. The main interactions among the five compartments, water, phytoplankton, zooplankton, detritus and superficial sediment, are illustrated in Fig. 2. Primary producers are represented by a single state variable, phytoplankton, whose dynamic is driven by water temperature, light intensity and DIN and DIP concentrations, and is affected by the grazing processes. The elementary composition of the plankton is assumed to be constant, with $\mathrm{C}: \mathrm{P}$ and $\mathrm{C}: \mathrm{N}$ equal to the Redfield ratio. Phytoplankton growth and nutrient uptake are coupled and described by a single-step kinetic. The actual specific growth rate is computed by using the so-called multiplicative model: the maximum specific growth rate constant, $\mu$ max, which depends on water temperature, is multiplied by four dimensionless factors, each ranging from zero to one. Each factor describes the limitation to the growth due to sub-optimal levels of light intensity, water temperature, DIN and DIP concentrations. The N and $\mathrm{P}$ limitations are computed in agreement with a standard Monod kinetic. The Steele formulation is used for describing the effect of the light intensity. The empirical relationship proposed by Lassiter and Kearns is used for modeling the 
effect of water temperature on the maximum growth rate, $\mu \max (\mathrm{T})$. Zooplankton is also described by means of a single state variable, which is representative of the pool of species which prey upon the phytoplankton. Grazing is described by a hyperbolic relationship (Holling II), while mortality, exudation and respiration of both phytoplankton and zooplankton are described as first order-processes: the kinetic constants exponentially increase with water temperature, in agreement with the Q10 relationship.

The detritus compartment is described by three state variables, in order to take into account separately the $\mathrm{C}, \mathrm{N}$ and $\mathrm{P}$ fractions. Detritus sinks and eventually enters the surface sediment box, which is also described by three state variables. The remineralization rate of the organic matter, which takes place both in the water column and in the surface sediment, depends on temperature and dissolved oxygen availability. This process involves the consumption of Dissolved Oxygen and leads to the release of nutrients back to the water column. Phosphorus is remineralized more rapidly than nitrogen and denitrification is also taken into account. The set of equations which describes the biological reaction is reported in Solidoro et al. [1], together with an indication of the set up of the model.

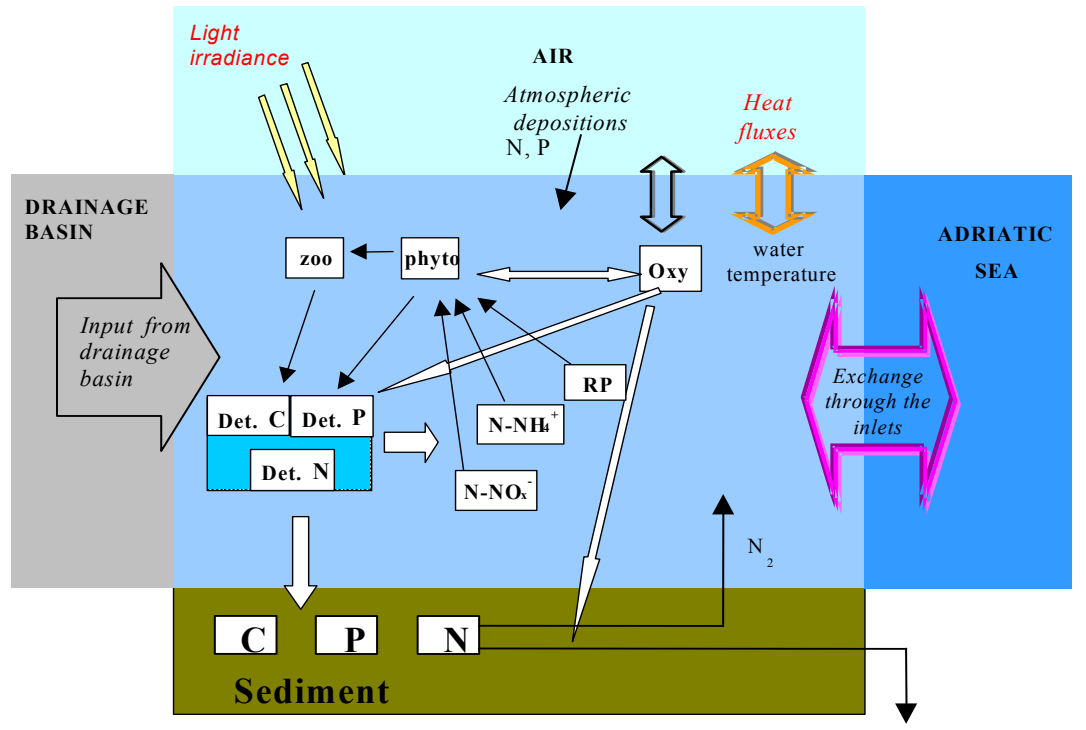

Figure 2: $\quad$ Main interactions among state variables (from Solidoro et al. [1]).

\section{Model applications}

\subsection{Managing fishing and rearing of Manila clam (Tapes philippinarun)}

The management of fishery and aquaculture activities is an important piece of Coastal Zone Management, since it represents a classical example of exploitation of renewable resources, subjected to both economic and ecological constrains. In 
fact, it depends heavily on water quality and trophic level, and it can be the basis of an important economic activity. Tapes philippinarum, commonly known as the Manila clam, was imported into the Northern Italian Adriatic lagoons at the beginning of the 1980s. It was soon reared in several lagoons of the northern Adriatic Sea, where local authorities set up aquaculture regimes which gave good socio-economic returns, but in the lagoon of Venice authorities feared that the harvesting of manila clams could have damaged other - already settled economic activities (Melaku Canu et al. [5]), and called for an environmental impact assessment of this activity before it was permited. A commercial exploitation started, with no defined regulation, nonetheless and grew exponentially so that by the beginning of the 90 s the lagoon of Venice became the most important site in terms of annual production and in terms of people involved in this activity (Melaku Canu et al. [5]). For over a decade fishing of clams continued on an open access basis, a policy which, as it is well known, rarely is the most efficient way to use a resource, and usually causes suboptimal situations, both from the ecological and economic points of view. In addition, since the fishing activity was carried on illegally, and without control or regulations, many other concerns arose. A list of problems included: use of illegal fishing tools, which heavily damage the bottom; health risks, for no certification could be given that the product was caught in healthy and not polluted sites; risk of overfishing; decreasing of the price of the goods, because of high availability of stock in the market; high social cost which could not be internalized. In short, the need for a better management of the resource was clear, both for the optimization of the clam market and to preserve the ecosystem (Melaku Canu et al. [5]).

This awareness prompted a number of studies, which included: a) the definition of the individual growth and population dynamic models concerning T. philippinarum, (Solidoro et al. [6]; Solidoro et al. [7]); b) the use of these models, in order to compare the ecological consequences and the revenues of uncontrolled open access strategies, controlled fishing activities (fixed quotas and fixed harvesting effort policies) and culture based fishery regimes (Solidoro et al. [7]). The results show that culture based fishery is to be preferred to a regulated type of fishing activity, and that ecologically conservative strategies (in which only large individuals are caught and always by using low efficiency fishing devices) are also more remunerative in the long run; c) the characterisation of the socio-economic context, in order to assess: i) the economic income the local community received from this activity, ii) how many people were involved and where they lived, iii) a first estimate of the total rearing area $\left(3 \times 10^{7} \mathrm{~m}^{2}\right)$ which could support a production comparable to that obtained in the open access fishing regime if a culture based fishery regime were implemented (Melaku Canu et al. [5]). The results of these complementing studies were integrated in the 3D finite difference coupled water-quality transport model (Pastres et al. [8]). The integrated model was then used to evaluate trophic potentialities of different areas of the water basin in terms of phytoplanktonic production and - by relating them to average energy requirements of Tapes Philippinarum and to other external constraints, such as 
healthiness and water column depth - to provide spatial distribution of a habitat suitability index for this species (fig 3). The locations of the lots to be devoted to clam rearing were then identified, by partitioning the total required rearing surface $\left(3 \times 10^{7} \mathrm{~m}^{2}\right)$ in keeping with the current spatial distribution of the fishing effort and in accordance with the criteria outlined above. The economical yields from different strategies of seeding density were then computed and compared. The model results indicate that revenues of optimised rearing/culture based fishery regimes in the selected areas would be similar to estimated yield in 1998, but the environmental impact would be much lower. The results of the selection process compare quite well with the spatial distribution of the areas which the Local Authorities assigned to the T. philippinarum fisheries in 2001.

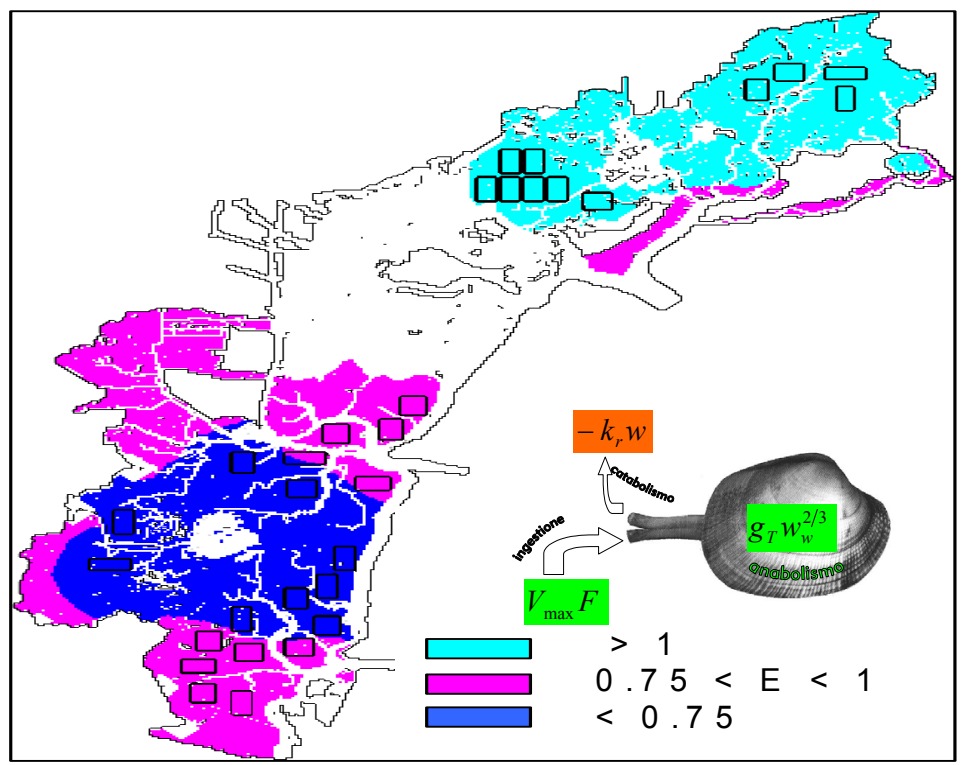

Figure 3: Distribution of aquaculture fields (boxes), in agreement with results from habitat suitability model (colours). See text for explanation.

\subsection{Determination of Maximum Permissible Loads}

In 1999 the Italian government issued an innovative law for the regulation of pollutant loads in water-bodies, which was based on the concept of Maximum Permissible Loads or Total Daily Maximum Load. Accordingly, Local Authorities were entrusted to make an inventory of the sources of pollution, and then to fix the level of emission of each activity, in order to maintain the concentrations of potentially dangerous substances below prescribed thresholds, called "quality targets", or "quality standards". The implementation of this policy may clearly benefit from the use of mathematical models, which can be used as tools for both estimating the Maximum-Permissible-Loads (MPLs) compatible with QTs, and exploring the consequences of different input 
scenarios. In fact, in mathematical terms, the loads are specified by a set of boundary conditions: numerical models can then be used for determining a functional relationship between the set of parameters which specify the boundary conditions and the output variables which one decides to compare with the Quality Targets. Once this task has been accomplished, one can solve the socalled "inverse problem", i.e. invert the function in order to estimate the MPLs which are compatible with the targets. This possibility was illustrated by using the lagoon of Venice as a case-study and the local sensitivity analysis in respect of each source of pollution as a tool in Pastres et al. [9]. In this way, together with the model trajectory, one can compute the "sensitivity matrix", which embodies the relevant information about the temporal evolution of the sensitivities and enable one to quickly estimate the perturbation on given output which would be caused by small variations of a subset of parameters around their nominal values. Two possible alternative definitions of Dissolved Inorganic Nitrogen QT were hypothesized. DIN MPL was computed when assuming that the QT had to be compared to the yearly averaged DIN concentration, which was found to be linearly related to the nutrient load. Furthermore, the sensitivity matrix was used for exploring the relationship between DIN MPL and the percentage of the water basin into which the yearly average value lay below QT. Such a relationship was found to be highly non-linear for percentages higher than $70 \%$, indicating that, in this range, small reductions in the nutrient loads would cause a great benefit in terms of compliance with the current legislation. Model results also indicated that a management policy based on the reduction of nutrient loads from specific sources would be more efficient than a general reduction of all sources. The main result of the paper, however, probably lies in the fact that it offers an example of how the methodology illustrated might be used for assisting policy makers.

\subsection{Effects of temporary closures of the inlets}

One of the major debates about the safeguard of Venice concerns the periodic flooding of the city, in concomitance of an exceptionally, but regularly occurring, high tide (acqua alta - 'high water'). As is well known, in the last decades the flooding events have increased in frequency and intensity, and several projects to prevent or at least mitigate consequence of 'high water' have been proposed.

One of the projects is the temporary closure of the inlets, employing a mobile gate specifically designed, in concomitance with the very high tide events. The importance and the complexity of such a project gave rise to a vast debate, still open, about the opportunity and the consequence of this solution. During the Environmental Impact Assessment (EIA) procedure, opponents of the operation stated that the closures could have a severe impact on the water quality of the lagoon, if implemented too frequently or for too long a time (Decree of the Ministry of the Environment, 24 December 1998). The underlying concern was that, without exchanging its water with the Adriatic Sea, the lagoon may no longer be able to self-purify its water at a rate high enough to cope with the input of pollutants from the historical city and the drainage basin. 
This hypothesis has been tested, under several simplifying assumptions, in a paper by Melaku Canu et al [10]. In the paper, which represents one of the very few examples of a paper in peer reviewed scientific literature dealing with aspects related to the MOSE problems, several scenarios of hydrodynamics and pollutant loads are considered, and their effects on BOD-DO concentration are compared.
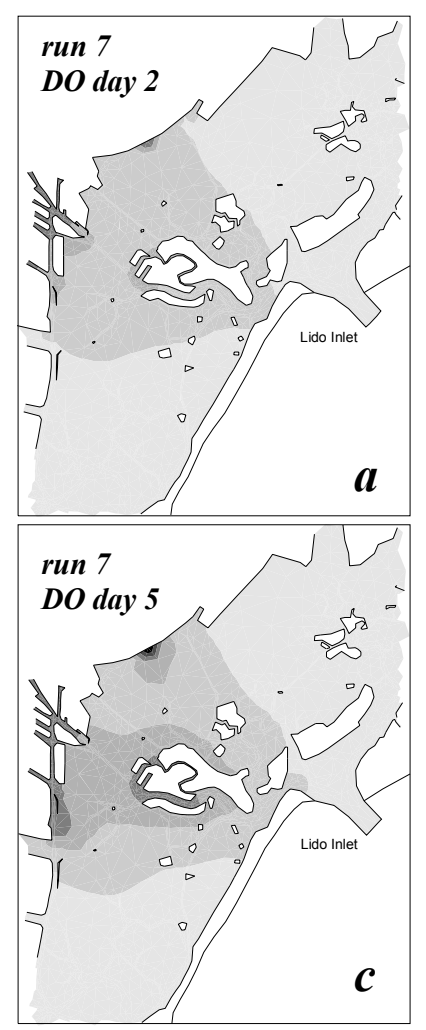
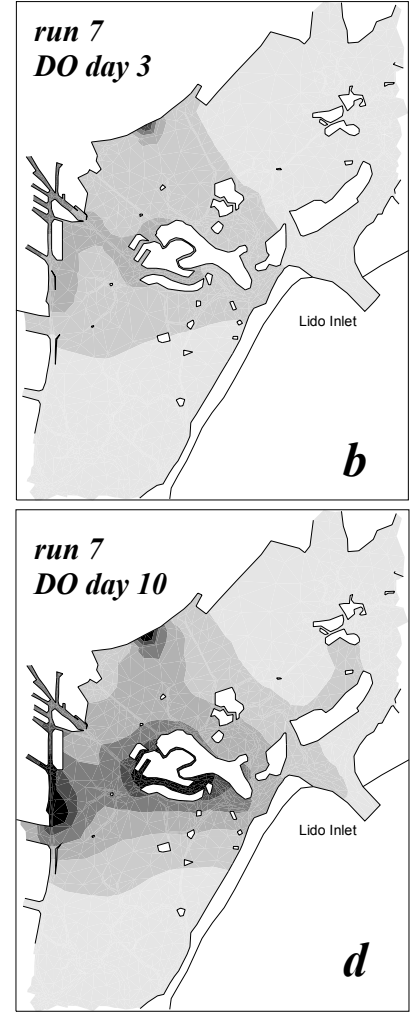
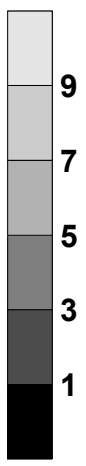

DO

$\mathrm{mg} / \mathrm{L}$

Figure 4: Spatial distributions of Dissolved Oxygen in 4 moments of the run 7 , in which the inlets are kept continuously closed (from Melaku Canu et al. [10]).

Numerical simulations are performed by using VELFEEM, a model developed by coupling some modules of the WASP5 code released by the United States Environmental Protection Agency and a primitive equation finite element hydrodynamic model, FEM, developed for the lagoon of Venice some years ago. The resulting structure gives the possibility to analyse the DO-BOD (biochemical oxygen demand) dynamics under winter conditions, in both the absence and the presence of a temporary closure of the inlets. The exercise could provide some suggestions about the effects of the closure on water quality. 
The simulations performed suggest that these closures do not significantly affect the water quality of the lagoon. In fact, even if the pollutant loads from industrial, agricultural, and domestic sources are doubled, in respect with the averaged winter values actually estimated, BOD concentrations are only slightly higher than the value which would be obtained without the temporary closures, and DO concentration never reaches very low levels. In any case the system seems to be able to recover rather easily to unperturbed situations (identified as the ones obtained by running the 'control' simulation with no closure at the inlets) when the inlets are opened again. As far as the DO-BOD dynamics is concerned, repeated temporary closures are not a major cause of critical conditions. Very low levels of DO concentration are instead reached when the inlets are kept continuously closed for very long tidal periods (more than 5 days), a scenario extremely unlikely or, any case, avoidable. Moreover, simulations show that the variability of the water quality is higher in response to the changes in loading factors than in response to the short term hydrodynamic reductions.

This paper, far from being a green light to the MOSE, which is a very important intervention with multifaceted aspects and which has to be considered from a much larger and more multidisciplinary perspective, shows that numerical models might be extremely useful for testing different scenarios, and reminds us that the discussion about the environmental impact of interventions aimed at solving the flooding problem should lose the character of a political debate, and became again the field for technological challenges and scientific confrontations.

\section{References}

[1] Solidoro C., Pastres R., Cossarini G., Ecol. Mod 2005.

[2] Solidoro, C., D. Melaku Canu, A. Cucco, G. Umgiesser, J Mar Sys, 51(1-4) 147-1602004.

[3] Ravera, O., J. Limnol. 59 19-30 2000.

[4] Umgiesser G., D. Melaku Canu, A. Cucco, C. Solidoro, J Mar Sys, 51(1-4) 123-146, 2004.

[5] Melaku Canu D., Solidoro C., Pastres R., Umgiesser G. Selected proceedings International conference sustainable development, Oporto, Portugal

[6] Solidoro C. , Pastres R., Melaku Canu D., Pellizzato M., Rossi R., Mar Ecol Prog Series, 199: 137-148. 2000.

[7] Solidoro, C., Melaku Canu, D. Rossi, R., Ecol. Mod 170 (2-3): 303-319. 2003

[8] Pastres, R., Solidoro, C., Cossarini, G., Melaku Canu, D. and Dejak, C., Ecol. Mod, 138(1-3):213-245. 2001.

[9] Pastres R., S. Ciavatta, G. Cossarini and C. Solidoro. Rel Eng \& Sys Saf, 79(2): 239-244 2003.

[10] Melaku Canu D., G. Umgiesser and C. Solidoro. Ecol. Mod., 138, (1-3): 215-230 2001. 